EPJ Web of Conferences 71, 00147 (2014)

DOI: $10.1051 /$ epjconf / 20147100147

(C) Owned by the authors, published by EDP Sciences, 2014

\title{
CERN and the high energy frontier
}

\author{
Emmanuel Tsesmelis ${ }^{1}$
}

${ }^{1}$ CERN, CH-1211 Geneva 23 Switzerland

\begin{abstract}
This paper presents the particle physics programme at CERN at the highenergy frontier. Starting from the key open questions in particle physics and the largescale science facilities existing at CERN, concentrating on the Large Hadron Collider (LHC), this paper goes on to present future possibilities for global projects in high energy physics. The paper presents options for future colliders, all being within the framework of the recently updated European Strategy for Particle Physics, and all of which have a unique value to add to experimental particle physics. The paper concludes by outlining key messages for the way forward for high-energy physics research.
\end{abstract}

\section{Key questions of particle physics}

Furthering our understanding of key questions in particle physics has relied on the interplay and synergy between various tools, such as accelerators, cosmic-ray detectors and reactors, and at various facilities, such as at a lepton collider, hadron collider and lepton-hadron collider, at both the energy frontier and the intensity frontier. The synergy has helped to elucidate the nature of electroweak symmetry breaking, the origin of mass and the discovery of the Higgs boson(s), which is a very central question in particle physics. This interplay will also continue to be important in extending our knowledge in other areas, such as the unification of forces, the fundamental symmetry of forces and matter, the number of space-time dimensions and the understanding of dark matter and dark energy. For many of these areas of research, new particles should appear at the Tera-electronvolt scale (TeV) or below and to provide better insight, the development of particle accelerators and particle detectors needs to proceed together with advanced theoretical calculations.

\section{The Large Hadron Collider}

The LHC is a 27-km circumference accelerator and is the largest scientific instrument ever built (see Figure 1). More than 10000 people have been involved in its design, construction and exploitation and the LHC and its experiments are prime examples for global collaboration. The start of physics exploitation for the LHC in 2010 ushered in a new and exciting era in fundamental science.

By colliding unparalleled high-energy and high-intensity beams, the LHC is opening up previously unexplored territory at the $\mathrm{TeV}$ scale in great detail, allowing the experiments to probe deeper inside matter and providing an understanding of processes that occurred very early in the history of the Universe.

This is an Open Access article distributed under the terms of the Creative Commons Attribution License 2.0, which permits unrestricted use, distribution, and reproduction in any medium, provided the original work is properly cited. 


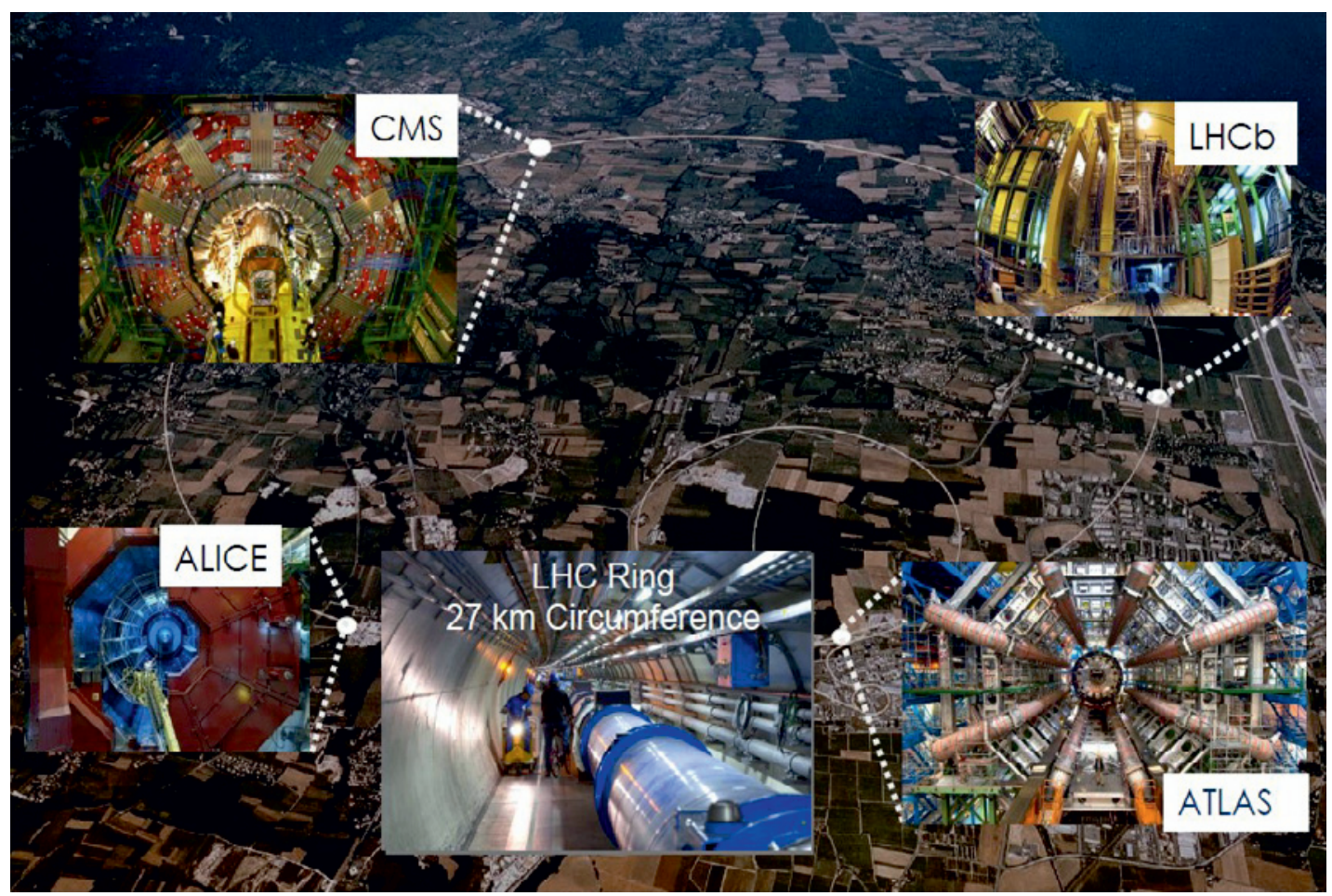

Figure 1: The LHC accelerator and experiments.

The LHC is primarily a proton-proton collider designed to operate at an unprecedented centre-of-mass energy of $14 \mathrm{TeV}$ and with a nominal luminosity of $1034 \mathrm{~cm}-2 \mathrm{~s}-1$. The LHC also collides heavy-ions (lead-lead) and has also ran in proton-lead collision mode.

The high $40 \mathrm{MHz}$ proton-proton collision rate and the tens of interactions per crossing result is an enormous challenge for the detectors and for the collection, storage and analysis of the data. An international collaboration, the Worldwide LHC Computing Grid (WLCG), has been created to distribute and analyse the vast amount of data produced at the LHC.

Most recently, 2013 was another excellent year of many achievements for the LHC. The year saw the successful completion of the first LHC physics running period (Run I) and the start of the first Long Shutdown (LS1) to prepare the LHC machine, injectors and experiments for operation up to $14 \mathrm{TeV}$ centre-of-mass energy at the design luminosity and also in view of the High Luminosity LHC (HLLHC). This outstanding performance is due to the increased experience in operating the LHC complex, including the injector chain, and to the beneficial impact of enhanced maintenance and consolidation efforts.

\section{The discovery of the Higgs boson}

A giant leap for science was made in 2012 with the discovery of the Higgs boson. The Higgs particle was discovered by the ATLAS and CMS collaborations, each of which involves over 3000 people from around the world. In the meantime, the scientific highlight for 2013 was the verification that this newly discovered particle by ATLAS and CMS in 2012 is a Higgs boson. This culminated in the award of several related prizes, most notably the Nobel Prize in Physics 2013 and the 2013 Prince of Asturias Award for Technical and Scientific Research. 
This completes the Standard Model of Particle Physics, which describes about 5\% of the mass-energy density of the Universe. The ATLAS and CMS experiments have also shown results of studies improving and consolidating our understanding of the Standard Model of Particle Physics, through further precision measurements of the inclusive jet cross-section, the single- and double- vector boson production, and the top quark. These experiments have also made extensive searches for new physics beyond the Standard Model (e.g. supersymmetry models).

\section{The European strategy for particle physics}

European particle physics is founded on strong national institutes, universities and laboratories, working in conjunction with CERN. The increased globalisation, concentration and scale of particle physics require a well-coordinated European strategy. This is a strategy of Europe for particle physics, it is a strategy in a global context and it is a strategy developed with international input. The process started with the adoption of the first strategy in 2006 and it has been recently updated in 2013.

The recently updated strategy contains four high-priority items for large-scale scientific activities. These scientific activities require significant resources, sizeable collaborations and sustained commitment.

1) The discovery of the Higgs boson is the start of a major programme of work to measure this particle's properties with the highest possible precision for testing the validity of the Standard Model and to search for further new physics at the energy frontier. The LHC is in a unique position to pursue this programme. Europe's top priority should be the exploitation of the full potential of the LHC, including the high-luminosity upgrade of the machine and detectors with a view to collecting ten times more data than in the initial design, by around 2030. This upgrade programme will also provide further exciting opportunities for the study of flavour physics and the quark-gluon plasma.

CERN has a programme at the energy frontier with the LHC for at least twenty years. This includes operation at centre-of-mass energies of up to the design $14 \mathrm{TeV}$ at the design luminosity and also at higher luminosities. The year 2013 saw the continuation of the performance-improving consolidation programme and of $\mathrm{R} \& \mathrm{D}$ for the HL-LHC aimed at realising the ambitious longer-term plans to provide a total integrated luminosity of the order of $3,000 \mathrm{fb}^{-1}$ (recorded) by the end of the life of the LHC. The HL-LHC was underlined as being a high-priority activity in the update of the European Strategy for Particle Physics. The HL-LHC phase will also entail the need to implement modifications to components in the insertion regions of the machine whose performance will have deteriorated due to radiation effects, such as the inner triplet quadrupole magnets. HL-LHC installation is planned for Long Shutdown 3 (LS3), scheduled for the period 2023-2025. Upgrades of the accelerator complex, detectors and WLCG are vital to fully exploit the full physics potential of the LHC.

2) To stay at the forefront of particle physics, Europe needs to be in a position to propose an ambitious post-LHC accelerator project at CERN by the time of the next Strategy update, when physics results from the LHC running at $14 \mathrm{TeV}$ will be available. CERN should undertake design studies for accelerator projects in a global context, with emphasis on proton-proton and electron-positron highenergy frontier machines. These design studies should be coupled to a vigorous accelerator $R \& D$ programme, including high-field magnets and high-gradient accelerating structures, in collaboration with national institutes, laboratories and universities worldwide.

In order to continue with searches and studies of new physics phenomena beyond the LHC, CERN is investigating possibilities for higher-energy hadron-hadron colliders. Possible proton-proton machines 
of higher energy than the LHC include the High-Energy LHC (HE-LHC) in the present LHC tunnel, roughly doubling the centre-of-mass energy of the LHC, and a Very-High Energy LHC (VHE-LHC) shown in Figure 3 and which has subsequently been names the Future Circular Collider (FCC), aimed at reaching a centre-of-mass energy up to $100 \mathrm{TeV}$ in a new circular

80-100 km tunnel. The main challenge for these machines is the availability of the high-field magnets. For the HE-LHC, the dipole design will profit from the R\&D needed for the high-gradient quadrupoles for HL-LHC. First studies on a new 80-100 km tunnel in the Geneva area have started and a kick-off workshop was held at the University of Geneva in February 2014. The FCC includes a machine with a centre-of-mass energy of $80 \mathrm{TeV}$ with $16 \mathrm{~T}$ dipole magnets based on $\mathrm{Nb}_{3} \mathrm{Sn}$ coils and a $100 \mathrm{TeV}$ centre-of-mass machine based on $20 \mathrm{~T}$ high-temperature superconducting dipole magnets.

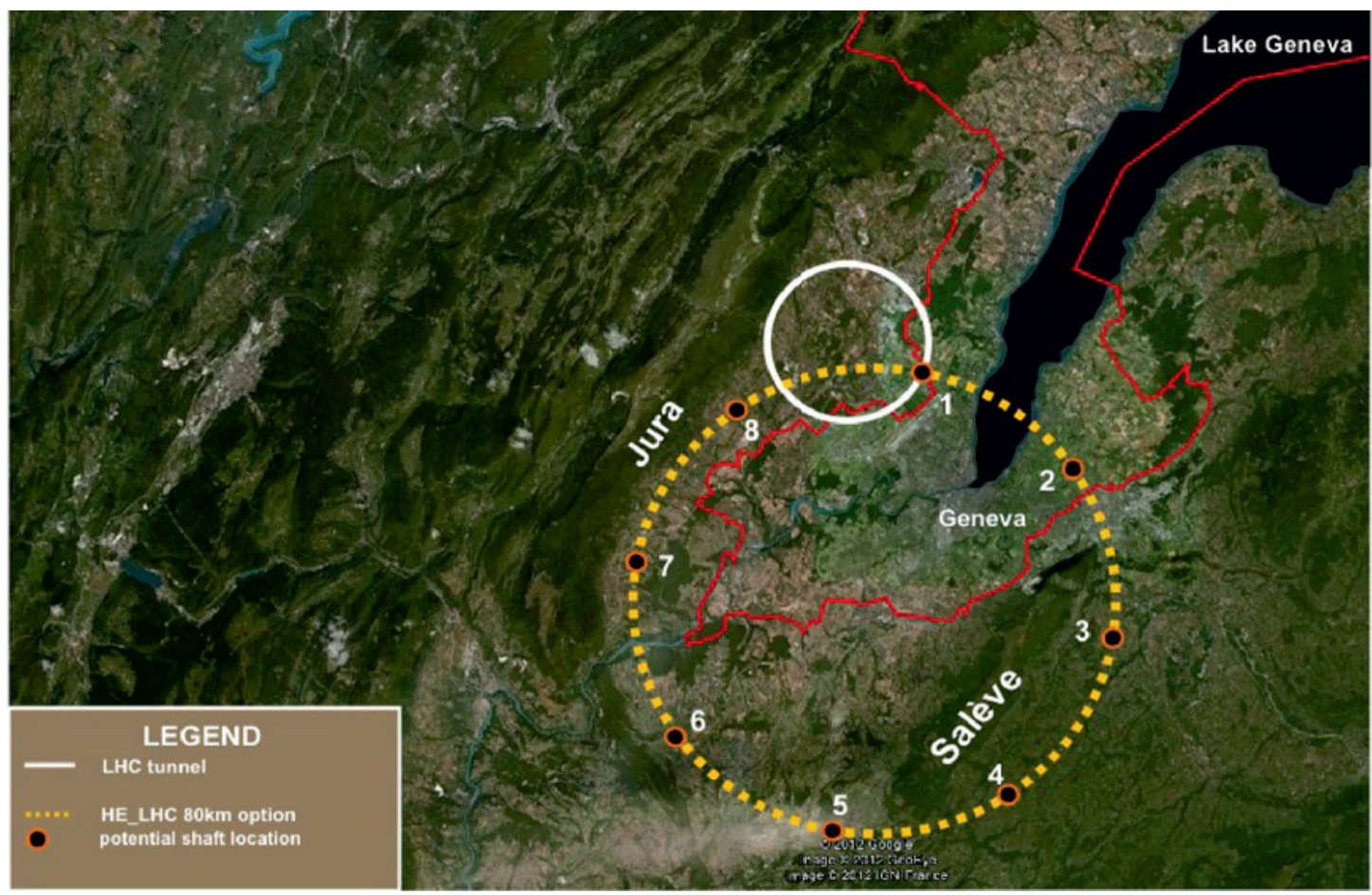

Figure 3: Possible location, based upon a geological study, of an 80-km ring for the FCC.

Such a large tunnel would also allow electron-positron collisions, such as the TLEP machine, roughly tripling the energy of the former LEP electron-positron collider, and electron-proton collisions in addition to proton-proton collisions. Studies and R\&D for high-field magnets as well as high gradient and high beam power superconducting RF cavities for the HL-LHC, HE-LHC, FCC (and TLEP) will enhance common efforts and exploit synergies.

Options for linear electron-positron colliders include the International Linear Collider (ILC) and the Compact Linear Collider (CLIC). CLIC, shown in Figure 4 and for which a Conceptual Design Report has already been published, could, in stages, reach a centre-of-mass energy up to $3 \mathrm{TeV}$. R\&D for CLIC, for example in high-gradient accelerating structures, continues for both the accelerator and detector in the framework of the Linear Collider effort and the CLIC Collaboration.

The CLIC Test Facility (CTF3) measurements have further established key items for the CLIC accelerator concept, including the two-beam acceleration scheme with accelerating gradients exceeding $100 \mathrm{MeV} / \mathrm{m}$ within the required breakdown rates for a $3 \mathrm{TeV}$ machine, and key performance parameters for alignment, stability and beam instrumentation. Increased effort has been 
directed at development of high-efficiency RF sources, modulators and klystrons, including studies and specifications towards procurement of prototypes. Common work with the ILC has continued.

The CLIC detector and physics study (CLICdp) was set up as an international cooperation with CERN as the host laboratory. Twenty institutes had subscribed by the end of 2013. CLICdp work focuses on hardware R\&D and software development for simulation and reconstruction. CLICdp collaborates closely with the detector and physics groups of the ILC.

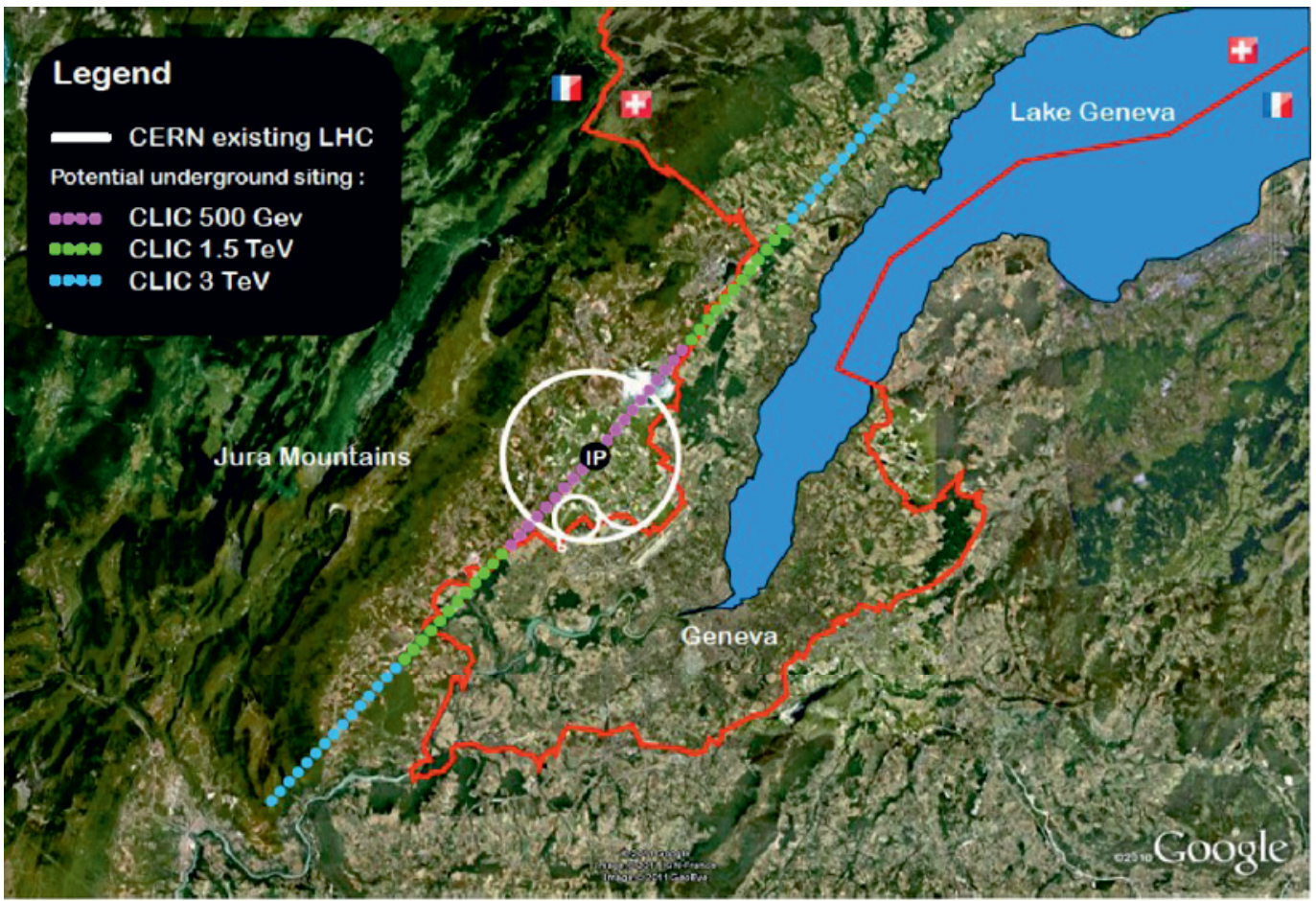

Figure 4: Potential siting of CLIC accelerator.

3) There is a strong scientific case for an electron-positron collider, complementary to the LHC, that can study the properties of the Higgs boson and other particles with unprecedented precision and whose energy can be upgraded. The Technical Design Report of the International Linear Collider (ILC) has been completed, with large European participation. The initiative from the Japanese particle physics community to host the ILC in Japan is most welcome, and European groups are eager to participate. Europe looks forward to a proposal from Japan to discuss a possible participation.

The best candidate for a lepton collider in the near future is the ILC, if it can be built soon. The ILC might start operations at a centre-of-mass energy of $250 \mathrm{GeV}$, and it should be upgradeable to $500 \mathrm{GeV}$ and with a possible extension to $1 \mathrm{TeV}$. The ILC is based on a mature design and the Japanese community has submitted to the Japan Government a request to host it. At CERN, the ILC efforts continue in the framework of the Linear Collider organisation structure and CERN will host the Linear Collider Collaboration (LCC) Directorate.

4) Rapid progress in neutrino oscillation physics, with significant European involvement, has established a strong scientific case for a long-baseline neutrino programme exploring $\mathrm{CP}$ violation and the mass hierarchy in the neutrino sector. CERN should develop a neutrino programme to pave the 
way for a substantial European role in future long-baseline experiments. Europe should explore the possibility of major participation in leading long-baseline neutrino projects in the US and Japan.

CERN aims to develop a neutrino programme to pave the way for a substantial European role in a future long-baseline experiment. To this end, CERN has started a platform to enable large-scale detector development, including WA104 (refurbished ICARUS T600 module), WA105 (R\&D on twophase LAr prototype) and tests with neutrino detectors as well as the study for a neutrino (test)beam in the North Area.

\section{Key messages on global projects}

In order to advance accelerator-based science, several factors come into play. Firstly, all projects need continuing accelerator and detector $\mathrm{R} \& \mathrm{D}$. Moreover, all projects need continuing attention concerning a convincing physics case, and to this end a close collaboration between experimental and theoretical physics is mandatory. There is a need to engage all countries with particle physics communities and to integrate particle physics from developing countries and regions. Expertise should be maintained across all regions and there is a need to ensure long-term stability and involvement across all regions.

\section{Conclusions}

In this paper, we have provided an account of global projects in high-energy physics. The paper presents current and future possibilities for such facilities, all within the framework of the recently approved update of the European Strategy for Particle Physics. The LHC and the new initiatives at the energy frontier will lead particle physics well into the next decades of fundamental research.

\section{Acknowledgements}

I would like to thank the organisers for the invitation to make this contribution and for the excellent organisation of the very interesting conference. 